\title{
ROBUST CONTROL SYSTEM DESIGN FOR SMALL UAV USING H2-OPTIMIZATION
}

\author{
Róbert SZABOLCSI \\ Óbuda University, Budapest, Hungary \\ szabolcsi.robert@bgk.uni-obuda.hu
}

\begin{abstract}
Unmanned aerial vehicles are famous for their wide range of applications. In D3 (Dirty-Dull-Dangerous) UAV applications flight conditions may vary on large scale. External disturbances like atmospheric turbulences and gusts may be subjected to UAV, and as a result, UAV flight mission might be conducted with high level of the degradation of the accuracy. Sensor noises are also present, and theirs negligence might lead to improper dynamic performances of the closed loop control systems. Uncertainties of the control systems being structured or unstructured may tend the closed loop control system to stability bounds. In worst case, uncertainties may destabilize closed loop control systems. The purpose of the author is to present a robust controller design method called H2-optimal design ensuring stability of the closed loop control systems with simultaneous dynamic performances predefined for the closed loop control system.
\end{abstract}

KEYWORDS: unmanned aerial vehicle, uncertainty, robust control, H2 optimal control

\section{Introduction}

The large scale variety of the unmanned aerial vehicle's flights (UAV) predicts worst case flight scenarios when UAV may interrupt flight mission and may return home to avoid critical flight situations. The UAV may have autopilot aboard ensuring stability and dynamic performances in total match with those of predefined ones. Besides external disturbances and internal sensor noises uncertainties may bring closed loop control system of the UAV to stability bounds, and as the worst case, uncertainties may destabilize the UAV closed loop automatic flight control system. UAV flights in real physical environment require a robust control system able to minimize, or, able to eliminate unwanted effects from disturbances, sensor noises, or, uncertainties.
The H2 controller design method is an extension of the LQG design procedure able to design state space controllers. The theoretical background and solution of theH2 controller design problem elaborated in the works of Bokor et al. (2014), Maciejowski (1989), Grimble (1994), Kwakernaak (2002), Dahleh et al. (1995), Vesely et al. (2015), and Weinmann (1991). Robust controller design applications are presented by Hartley (1990), Morari et al. (1991). Multivariable dynamical systems were investigated by Franklin et al. (1994), Friedland (1986), Golten el at. (1991), Ogata (1999), Skelton (1988). The aircraft spatial motion dynamics was exhaustively investigated by McLean (1990). The UAV longitudinal motion dynamics is presented by Szabolcsi (2016). The MATLAB R2017b (2017), and MATLAB Control 
System Designer 10.3 (2017), and Robust Control Toolbox have been used for design and for simulation purposes.
2. The LQG Control Problem Formulation

The block diagram of the random system can be seen in Figure no. 1 .

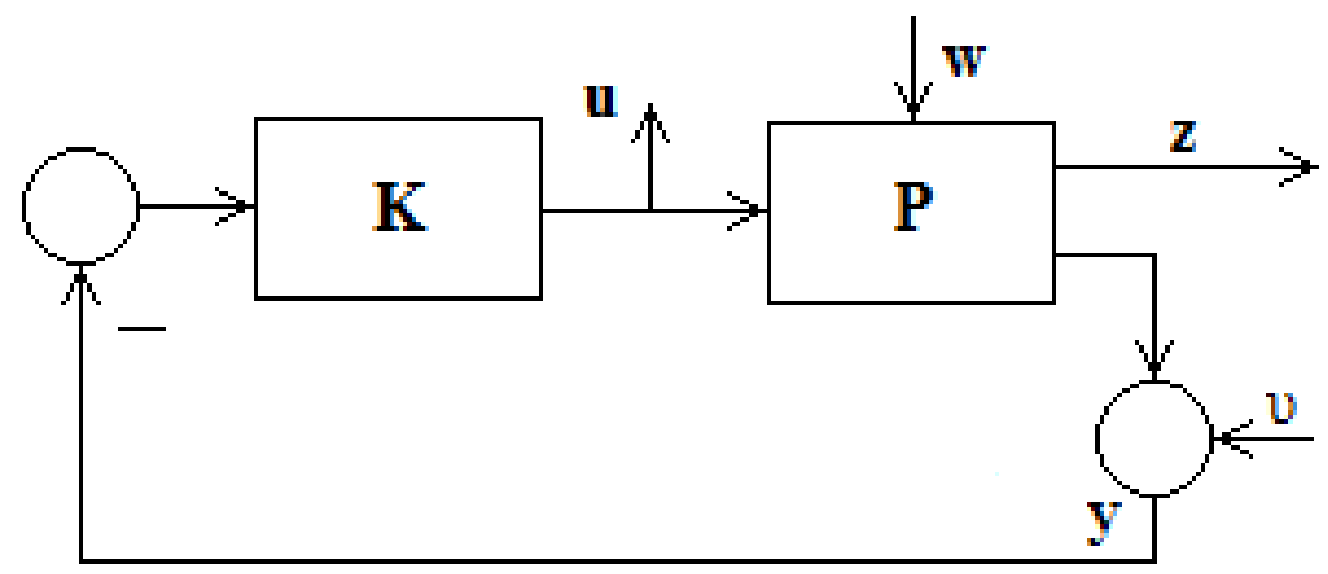

Figure no. 1: Block diagram of the LQG optimization system

The plant $\boldsymbol{P}_{\text {is }}$ derived by the state equation given below (Bokor, et al., 2014; Kwakernaak, 2002):

$$
\dot{\mathrm{x}}=\mathrm{A} x(t)+\mathrm{Bu}(t)+F w(\mathrm{t})
$$

where $w$ is the white noise model of the plant disturbance, $u$ is the control input vector, $y$ is the measured output vector. The regulated output vector $z$ (tracking errors, control inputs, measured outputs, states) and the measured output vector $y$ are defined as follows:

$$
\left.\begin{array}{c}
z(t)=D x(t) \\
y(t)=C x(t)+v(t)
\end{array}\right\}
$$

where $v$ represents the white noise of the sensors applied for measurement purposes.

The optimal LQG design allows findingclosed loop control system stabilizing controller Kthat will minimize the cost function of the form:

$$
\lim _{t \rightarrow \infty} E\left\{z^{T}(t) z(t)+u^{T}(t) u(t)\right\}
$$

where $E\{$.$\} is the expected value operator.$
Solution of this problem has been elaborated in the works of Bokor, et al. (2014), and Kwakernaak (2002). The basic idea of the solution to that problem is the well-known separation principle. In that framework, the optimal compensator is found in two steps, i.e. the stabilizing controller is the interconnection of the optimal Kalman filter, and, the optimal static state feedback. It means that controller synthesis requires solution to two algebraic Ricatti equations (ARE). The first ARE serves for the solution of the linear quadratic regulator problem, and, the second ARE is the one serving for the solution of the linear quadratic estimator problem. The solution of the LQG optimal controller problem requires a priori data about plant disturbances and sensor noises being considered.

It is well-known that the LQG problem can be generalized to that of the so-called standard problem, and, the stochastic interpretations may be eliminated. The standard configuration of the closed loop control system can be seen in Figure no. 2. 


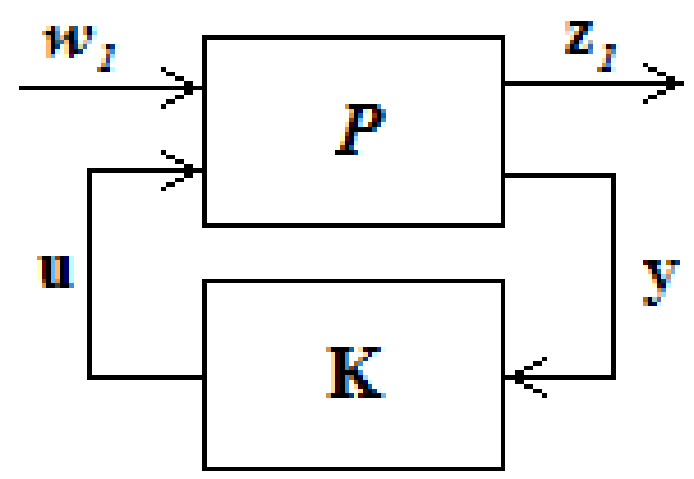

Figure no. 2: Closed loop system standard configuration

In Figure no. $2 P$ represents the so-called generalized plant model is as follows:

$$
\left[\begin{array}{l}
z \\
y
\end{array}\right]=P(s)\left[\begin{array}{l}
w \\
u
\end{array}\right]=\left[\begin{array}{ll}
P_{11}(s) & P_{12}(s) \\
P_{21}(s) & P_{22}(s)
\end{array}\right]\left[\begin{array}{l}
w \\
u
\end{array}\right](4
$$

where $z:=\left[\begin{array}{ll}z & y\end{array}\right]^{T}, w:=\left[\begin{array}{ll}w & u\end{array}\right]^{T}$, $\mathrm{u}$ is the control input vector, $w$ is the vector of the exogenous inputs (fixed commands, unknown commands, disturbances, noises), $z$ is the regulated output, and, finally, $y$ is the measured output vector. Partitions in $P(\mathrm{~s})$ in equation (4) can be expressed as (Kwakernaak, 2002):

$$
\left.\left.\begin{array}{l}
P_{21}(s)=\left[\begin{array}{cc}
D(s I-A)^{-1} F & 0 \\
0 & 0
\end{array}\right] ; P_{22}(s)=\left[\begin{array}{c}
\mathrm{D}(s I-A)^{-1} B \\
I
\end{array}\right] \\
P_{72}(s)=\left[\begin{array}{ll}
C(s I-A)^{-1} F & I
\end{array}\right] \mathrm{s} P_{n 2}(s)=\left[C(s I-A)^{-1} B\right]
\end{array}\right]\right\}
$$

The LQG system design problem can be solved if to minimize the steady-state value of the expected value of $E\left\{z^{T}(t) z(t)\right\}$. Using Figure no. 2 closed loop system regulated output $z$ can be expressed in the term of the Laplacetransform given below:

$$
z=T_{z_{1} w_{1}}(s) w
$$

where $\boldsymbol{T}_{z w}$ is the closed loop control system transfer matrix defined as:

$$
\begin{aligned}
& T_{z_{1} w_{1}}(s)=P_{11}(s)+P_{12}(s)[I- \\
& \left.K(s) P_{22}(s)\right]^{-1} K(s) P_{21}(s)
\end{aligned}
$$

If the sensor noise $v$ represents the white noise with unit intensity matrix of $I$, and, if the closed loop control system is stable one, then the integral performance index used for the causal controller design of $K(\mathrm{~s})$ can be derived using Parseval's formula as follows:

$$
\begin{aligned}
& \lim _{t \rightarrow \infty} E\left\{z^{T}(t) z(t)\right\}= \\
& \frac{1}{2 \pi} \operatorname{tr} \int_{-\infty}^{+\infty} T_{z_{1} w_{1}}(-j \omega) T_{z_{1} w_{1}}(j \omega) d \omega \rightarrow \\
& \text { Min }
\end{aligned}
$$

The right-hand side of the equation (8) can be rewritten as the square of the second norm of the closed loop control system stable transfer matrix $T_{z_{1} w_{1}}$ :

$$
\begin{aligned}
& \left\|T_{z_{1} w_{1}}(s)\right\|_{2}^{2}= \\
& \frac{1}{2 \pi} \operatorname{tr} \int_{-\infty}^{+\infty} T_{z_{1} w_{1}}(-j \omega) T_{z_{1} w_{1}}(j \omega) d \omega \rightarrow \\
& \text { Min }
\end{aligned}
$$

Summing up previous statements, solution of the LQG optimization problem outlined above represents the minimization of the second norm of the closed loop stable transfer matrix $T_{z_{1} w_{1}}$.

Solution of the problem outlined previously in time domain is more convenient. For further discussions it is assumed that the plant dynamics $P(\mathrm{~s})$ is expressed with the following state space representationelaborated and shown by Kwakernaak (2002): 


$$
\left.\begin{array}{c}
\dot{x}(t)=A x(t)+B_{1} w(t)+B_{2} u(t) \\
z(t)=C_{1} x(t)+D_{11} w(t)+D_{12} u(t) \\
y(t)=C_{2} x(t)+D_{21} w(t)+D_{22} u(t)
\end{array}\right\}
$$

The plant dynamics $P(s)$ can be represented by the following matrix partitions:

$$
P(s):=\left[\begin{array}{llll}
A & \| & B_{1} & B_{2} \\
-C_{1} & - & - & - \\
C_{2} & \| & D_{21} & D_{12} \\
D_{22}
\end{array}\right]
$$

In equation (11) $D_{11}=0$ in order to obtain a finite second norm defined by equation (9), and $D_{22}=0$ because real physical systems always have a zero gain at infinite frequency. System matrices in equation (10) are supposed to meet following assumptions:

1. the pair of matrices $\left(A, B_{2}\right)$ is stabilizable one;

2. $D_{12}^{T} D_{12}$ is invertible one;

3. $D_{12}^{T} C_{1}=0$;

4. the pair of matrices $\left(C_{1}, A\right)$ has no unobservable modes on the complex plain imaginary axis;

5. The pair of matrices $\left(C_{2}, A\right)$ is detectable;
6. $D_{21} D_{21}^{T}$ is invertible;

7. $D_{21} B_{1}^{T}=0$;

8. the pair of matrices $\left(A, B_{1}\right)$ has no uncontrollable modes on the complex plain imaginary axis.

The assumptions of (1-4) stand for the solution of the optimal state feedback problem, whilst assumptions (5-8) are related to the solution of the optimal state estimation problem.

\section{The $\boldsymbol{H}_{2}$-Optimization Problem}

The $\mathrm{H}_{2}$-optimization method removes the stochastics from the LQG optimization technique. The formulation and solution of the $\mathrm{H}_{2}$-optimization problem replaces the stochastic dynamical system LQG optimization with minimization of the second norm of the closed loop control system transfer matrix $T_{z_{1} w_{1}}$. The conventional LQG optimization can be considered for the special case of the $\mathrm{H}_{2}$-optimization problem.

The conventional LQG problem is the special case of the generalized LQG problem outlined and discussed using Figure no. 3.

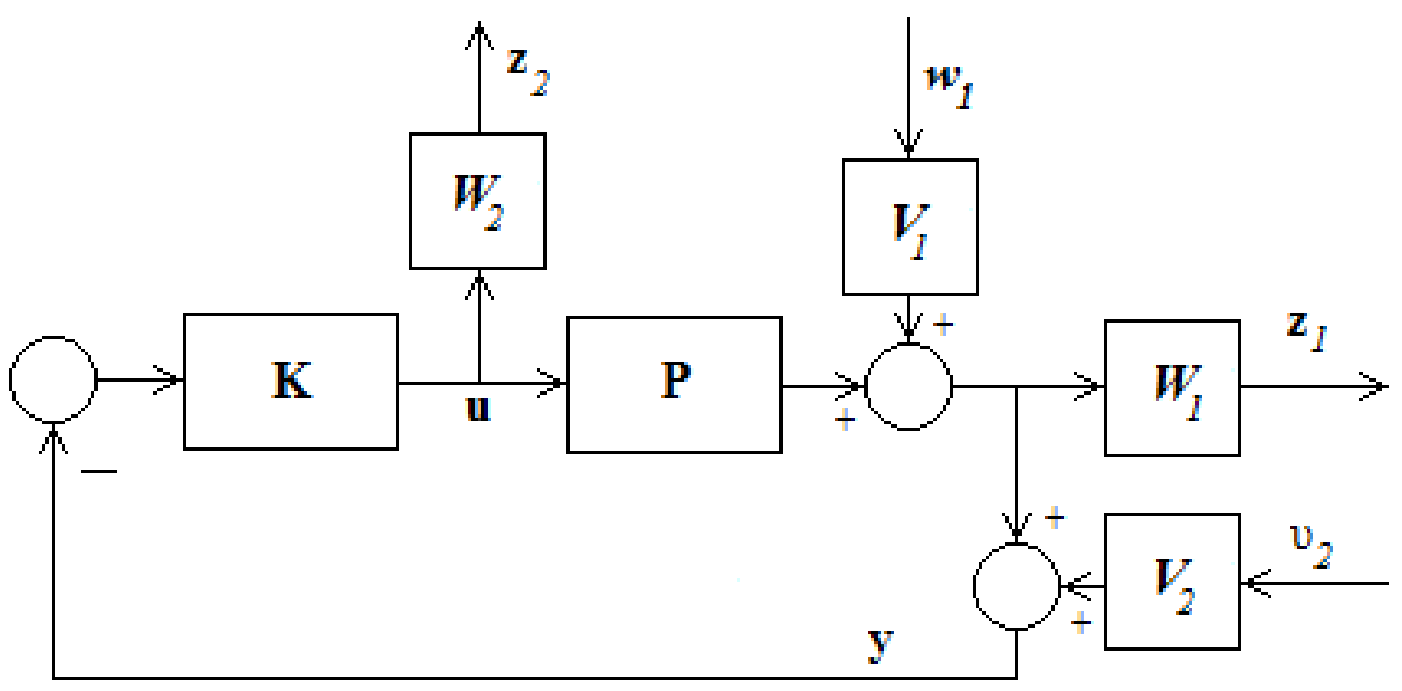

Figure no. 3: The generalized LQG problem - the $\mathrm{H}_{2}$ mixed sensitivity problem 
The colored disturbance $w_{1}(s)$, and the measurement noise $v_{2}(s)$ are passed through the shaping filters of $V_{1}(s)$ and $V_{2}(s)$ to determine theirs frequency content. The frequency domain weighting of the controlled output $z_{1}(s)$ and input $u(s)$ are determined by weighting functions of $W_{1}(s)$, and $W_{2}(s t)$, respectively.

The control system design based upon $\mathrm{H}_{2}$-optimization technique aimed at achieving following set of the criteria:

1. closed-loop system stability;

2. closed-loop system dynamic performances;

3. closed-loop system robustness.

To fulfill simultaneously system of criteria defined above the controller must ensure:

1. large open loop gains at low frequencies;

2. small open loop gains at high frequencies;

3. open loop gain is kept away from the critical point of $-1+0 j$ at the crossover frequencies.

For closed loop control system the sensitivity function of $S$, and, the complementary sensitivity functions of $T(\mathrm{~s})$ can be expressed using following equations:

$$
\left.\begin{array}{l}
S(s)=\frac{1}{I+P(s) K(s)} \\
T(s)=\frac{P(s) K(s)}{I+P(s) K(s)}
\end{array}\right\}
$$

In equation (12) the sensitivity function of $S(\mathrm{~s})$ determines how the disturbance effects on the closed loop control system output. The complementary sensitivity function of $T(\mathrm{~s})$ determines the closed loop system response. From equation it is easy to find out that:

$$
S(s)+T(s)=1
$$

Using functions $S(\mathrm{~s})$ and $T(\mathrm{~s})$ the design criteria explained above can be redefined as follows:

1. sensitivity function $S(\mathrm{~s})$ must be small at low frequencies;

2. complementary sensitivity function $T(\mathrm{~s})$ must be small at high frequencies;

3. avoid peaks in $S(\mathrm{~s})$ and $T(\mathrm{~s})$ at crossover frequencies.

To achieve those targets defined above let us select $V_{2}(s)=0$. Thus, we have:

$$
\begin{aligned}
& {\left[\begin{array}{l}
z_{1} \\
z_{2}
\end{array}\right]=\left[\begin{array}{l}
W_{1}(s) S(s) V_{1}(s) \\
W_{2}(s) R(s) V_{1}(s)
\end{array}\right] w_{1}(s)=} \\
& T_{z w}(s) w_{1}(s)
\end{aligned}
$$

where $R$ is the input sensitivity function and it is expressed as:

$$
R(s)=\frac{K(s)}{[I+P(s) K(s)]} ; T(s)=P(s) R(s)
$$

If the closed loop control system id the SISO one, minimization of the second norm of the system transfer matrix (closed loop complementary sensitivity function) $\boldsymbol{T}_{z w}$ means:

$$
\begin{aligned}
& J=\frac{1}{2 \pi} \int_{-\infty}^{+\infty}\left\{\left|W_{1}(j \omega) S(j \omega) V_{1}(j \omega)\right|^{2}+\right. \\
& \left.\left|W_{2}(j \omega) R(j \omega) V_{1}(j \omega)\right|^{2}\right\} d \omega \rightarrow \text { Min }
\end{aligned}
$$

To achieve design goals suitable selection of weights of $V_{1}(s), W_{1}(s)$, and $W_{2}(s)$ is required, and for that numerous considerations are available and widely applied in control engineering. The latter discussion will be about weight selection for the SISO control system case.

The choice of the filter $V_{1}(s)$ is often conducted leaning on LQG design problem, and we have:

$$
V_{1}(s)=C(s I-A)^{-1} F
$$


If to set $F=B$, we have:

$$
V_{1}(s)=P(s)=C(s I-A)^{-1} B
$$

where $P(\mathrm{~s})$ represents the open-loop plant transfer function.

The choice of the filter $V_{1}(s)$ can be rephrased as it will shape the frequency content of the disturbance according to the open-loop frequency response function. The filter $V_{1}(s)$ is often chosen to be (Kwakernaak, 2002):

$$
V_{1}(s)=P(s) \frac{s+\alpha}{s}
$$

In equation $\alpha$ is the design parameter. The pole at 0 in $V_{1}(s)$ ensures $S(0)=0$, and, it will lead to the finite second norm defined by equation (16).

If weighting function $V_{1}(s)$ is chosen to have integral action (pole at 0 ) filter $W_{1}(s)$ may serve for fine tuning. The safe initial selection is $W_{1}(s)=1$.

Selection of the filter $W_{2}(s)$ must have a zero at 0 so as to cancel corresponding pole of the filter $V_{1}(s)$. There are two widely applied transfer functions of the filter as follows:

$$
W_{2}(s)=\frac{\rho s}{s+\alpha}
$$

$$
\dot{x}=\left[\begin{array}{c}
\dot{v} \\
\dot{p} \\
\dot{r} \\
\dot{y}
\end{array}\right]=A x+B u=
$$

$\left[\begin{array}{cccc}-0,7724 & 0 & -18,9671 & 9,0867 \\ 1,9247 & -19,9149 & 7,7565 & 0,0000 \\ 69,1314 & -23,8689 & -2,5966 & 0,0000 \\ 0,0000 & 1,0000 & 0,0000 & 0,0000\end{array}\right]\left[\begin{array}{l}v \\ p \\ r \\ \gamma\end{array}\right]+\left[\begin{array}{cc}0,0000 & 2,2582 \\ -23,8289 & 1,5015 \\ -11,7532 & -15,2855 \\ 0 & 0\end{array}\right]\left[\begin{array}{l}\delta_{a} \\ \delta_{r}\end{array}\right]$

22)

Where $v$ is the lateral speed, $p$ is the roll rate, $r$ is the yaw rate, $\gamma$ is the roll angle, $\delta_{a}$ is the aileron deflection, $\delta_{r}$ is the rudder deflection, respectively. where filter parameters $\rho$ and $\alpha$ are used for fine tuning, and, $U(\mathrm{~s})$ is expected to have at least zero roll-off.

$$
W_{2}(s)=\frac{\rho s}{s+\alpha}(1+\tau s)
$$

where $U(\mathrm{~s})$ has minimal roll-off at $1 \mathrm{~dB} / \mathrm{dec}$.

\section{A Numerical Example for the UAV $\mathrm{H}_{2}$ Optimal Control System Design}

The broader range of the UAS is available the broader scale of the possible application is accessible. The UAV as a part of the UAS is becoming more and more complex. To ensure flight safety of the UAV equivalent or higher to that of the manned aircraft criteria the automatic flight control system or autopilot must be implemented aboard.

The UAV spatial motion can be modelled using MIMO, or SISO-approach. The MIMO dynamical model of the aircraft is given by McLean (1990). The Boomerang-60 Trainer small UAV lateral/directional motion MIMO dynamical model is given by P. Eng (2011) and by Szabolcsi (2016) and is as follows:

$$
\dot{p}(t)=-19,9149 p(t)-23,8289 \delta_{\alpha}(t)
$$


The transfer function of the Boomerang-60 UAV is as follows:

$Y(s)=\frac{p(s)}{-\delta_{a}(s)}=\frac{23,8289}{s+19,9149}=\frac{A}{1+s T}$

where $A=1,1965, T=0,0502 \mathrm{~s}$.

The roll rate stability augmentation system is often serves as inner loop in the roll angle stabilization of the UAV, and it can be seen in Figure no. 4.

The corresponding plant equations are as follows:

$$
\left.\begin{array}{c}
p_{r e f}=w \\
p=x_{1} \\
x_{2}=0,1 z_{1} \rightarrow z_{1}=10 x_{2} \\
\dot{x}_{1}=-\frac{1}{T} x_{1}+\frac{A}{T} u \\
\dot{x}_{2}=-x_{1}-10 x_{2}+w \\
z_{2}=2 u \\
y=-x_{1}+w
\end{array}\right)
$$

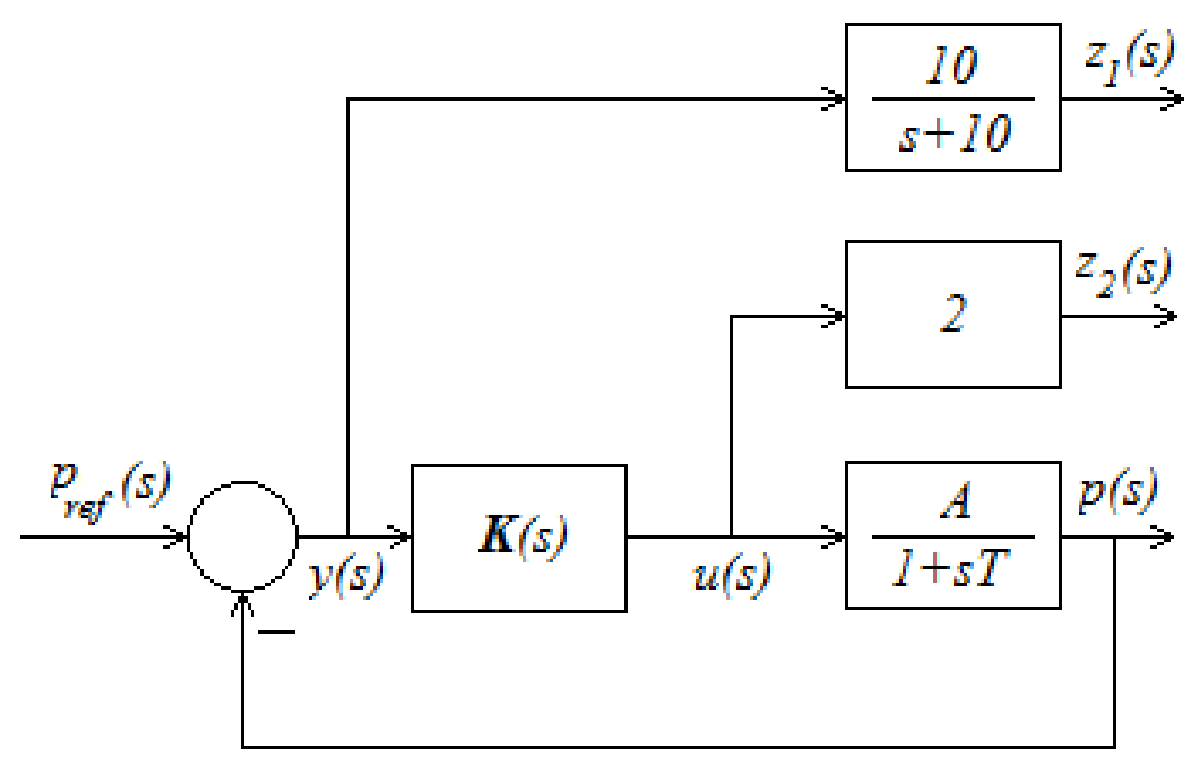

Figure no. 4: The roll rate stability augmentation system of the UAV

Using equation (25) the plant matrices defined by equation (11) can be derived as follows:

$$
\left.\begin{array}{c}
A=\left[\begin{array}{cc}
-\frac{1}{T} & 0 \\
-1 & -10
\end{array}\right] ; B_{1}=\left[\begin{array}{l}
0 \\
1
\end{array}\right] ; B_{2}=\left[\begin{array}{c}
\frac{A}{T} \\
0
\end{array}\right] \\
C_{1}=\left[\begin{array}{cc}
0 & 10 \\
0 & 0
\end{array}\right] ; C_{2}=\left[\begin{array}{ll}
-1 & 0
\end{array}\right] \\
D_{11}=\left[\begin{array}{l}
0 \\
0
\end{array}\right] ; D_{12}=\left[\begin{array}{l}
0 \\
2
\end{array}\right] ; D_{21}=1 ; D_{22}=0
\end{array}\right\}
$$

The TITO (Two inputs - Two outputs) closed loop system (Figure no. 2) plant dynamics has been constructed using mktito.m embedded function of MATLAB.
The optimal H2 state space controller K(s) has been designed using MATLAB and its toolboxes via minimizing the closed loop control system stable transfer matrix $\mathrm{T}_{\mathrm{z}_{1} \mathrm{w}_{1}}$ using integral performance index defined by equation (8). The h2syn.m embedded function of MATLAB will find:

K - LTI optimal controller;

$\mathrm{CL}=\operatorname{lft}(\mathrm{P}, \mathrm{K})-$ LTI closed loop control system transfer function $\mathrm{T}_{\mathrm{z}_{1} \mathrm{w}_{1}}$; $\gamma=\left\|\mathrm{T}_{\mathrm{z}_{1} \mathrm{w}_{1}}\right\|_{2}$.

Gam = norm (CL) $-\mathrm{H} 2$ optimal cost

Results of the $\mathrm{H} 2$ optimal controller synthesis are as follows below: 


$$
\begin{aligned}
& K=\left[\begin{array}{lll}
A_{k} & \mid & B_{k} \\
\hdashline & \frac{1}{C_{k}} & D_{k}
\end{array}\right] \\
& \left.A_{k}=\left[\begin{array}{cc}
-21 & 22,11 \\
0 & -10
\end{array}\right] ; B_{k}=\left[\begin{array}{l}
0 \\
1
\end{array}\right] ; C_{k}=\left[\begin{array}{ll}
-0,04536 & 0,9279
\end{array}\right] ; D_{k}=0\right) \\
& C L=\left[\begin{array}{lll}
A_{c l} & \mid & B_{c l} \\
\hdashline & \frac{1}{C_{c l}} & \multicolumn{1}{c}{D_{c l}}
\end{array}\right] \\
& A_{c l}=\left[\begin{array}{cccc}
-19,91 & 0 & -1,081 & 22,11 \\
-1 & -10 & 0 & 0 \\
0 & 0 & -21 & 22,11 \\
-1 & 0 & 0 & -10
\end{array}\right] \\
& \left.B_{c l}=\left[\begin{array}{l}
0 \\
1 \\
0 \\
1
\end{array}\right] ; C_{c l}=\left[\begin{array}{cccl}
0 & 10 & 0 & 0 \\
0 & 0 & -0,09072 & 1,856
\end{array}\right] ; \quad D_{c l}=\left[\begin{array}{l}
0 \\
0
\end{array}\right]\right) \\
& Y=\left\|T_{z_{1} w_{1}}\right\|_{2}=2,1972
\end{aligned}
$$

The UAV roll rate stability augmentation system has been tested in time domain. Results of the computer simulation can be seen in Figure no. 5.

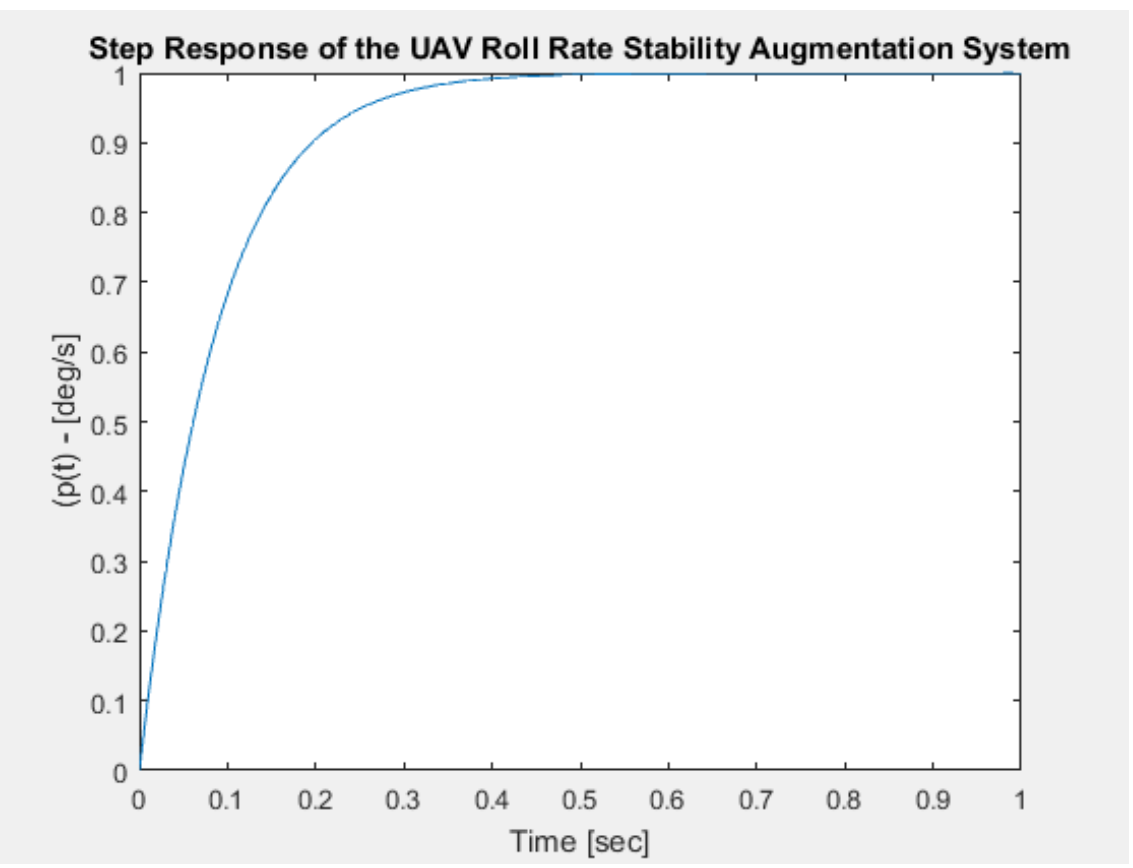

Figure no. 5: Time domain behavior of the UAV

From Figure no. 5 it is evident that UAV closed loop roll rate stability augmentation system has very fast response to the unit step change in the roll rate. There is no overshoot in the normalized step response, i.e., the entire closed loop control system behaves with non-oscillatory feature. 


\section{Conclusions}

The H2 optimal controller synthesis method is used long time very intensively. The controller design is supported by MATLAB, and the simulation is also easy-to-conduct using MATLAB toolboxes. The robustness of the UAV roll rate stability augmentation system is ensured.
The fast response in roll rate means ability of the UAV to change bank angle very fast, too. This will allow use very effectively the roll control channel if to conduct collision avoidance missions, or, if to execute emergency landing being powered or unpowered.

\section{REFERENCES}

Bokor, J., Gáspár, P., \& Szabó, Z. (2014). Modern Control Engineering. Budapest: Budapest University of Technology and Economics.

Dahleh, M. A., \& Diaz-Bobillo, I. J. (1995). Control of Uncertain Systems - A Linear Programming Approach. Englewwod Cliffs, New Jersey: Prentice-Hall.

Eng, P.C.S. (2011). Path Planning, Guidance and Control for a UAV Forced Landing. Queensland University of Technology.

Franklin, G. F., Powell, J. D., \& Emami-Naeini, A (1994). Feedback Control of Dynamic Systems. Reading - Menlo Park - New York - Don Mills - Wokingham Amsterdam - Bonn - Sydney - Singapore - Tokyo - Madrid - San Juan - Milan - Paris: Addison-Wesley Publishing Company.

Friedland, B. (1986). Control System Design. New York - London: McGraw-Hill.

Golten, J., \& Verwer, A. (1991). Control System Design and Simulation. New York: McGraw-Hill Book Company.

Grimble, M. J. (1994). Robust Industrial Control - Optimal Design Approach for Polynomial Systems. New York: Wiley.

Hartley, G. A. (1990). F-18 Robust Control design Using $\mathrm{H}_{2}$ and H-infinity methods. Monterey, CA: Naval Postgraduate School.

Kwakernaak, H. (2002). $\mathrm{H}_{2}$-Optimization - Theory and Applications to Robust Control Design. Annual Reviews in Control, 26, 45-56.

Maciejowski, J. M. (1989). Multivariable Feedback Design. Wokingham - Menlo Park - New York - Don Mills - Amsterdam - Bonn - Sydney - Singapore - Tokyo - Madrid San Juan: Addison-Wesley Publishing Company.

Morari, M., \& Zafiriou, E. (1991). Robust Process Control. Englewood Cliffs, NJ: PrenticeHall International, Inc.,

MathWorks. (2017). MATLAB R2017b, User's Guide. Natick, MA: Author.

MathWorks. (2017). MATLAB Control System Designer/Control System Toolbox 10.3, User's Guide. Natick, MA: Author.

MathWorks. (2017). MATLAB Control System Designer/Robust Control Toolbox, User's Guide. Natick, MA: Author.

McLean, D. (1990). Automatic Flight Control Systems. New York - London - Toronto - Sydney - Tokyo - Singapore: Prentice-Hall International Ltd.

Ogata, K. (1999). Modern Control Engineering. New York - London: Prentice-Hall.

Skelton, R. E. (1988). Dynamic Systems Control. NewYork - Chichester - Brisbane Toronto - Singapore: John Wiley \& Sons.

Szabolcsi, R. (2016). Automatic Flight Control of the UAV. Budapest: Óbuda University.

Vesely, V., Rosinova, D., \& Kozakova, A. (2015). Robust Controller Design. Bratislava: Slovak University of Technology in Bratislava.

Weinmann, A. (1991) Uncertain Models and Robust Control. Wien, New York: Springer-Verlag. 